\title{
Channel Selection Strategy of the Supply and the Demand of Service Based on Evolutionary Game
}

\author{
Bu Xinyi ${ }^{1, a}$, Dong Ying ${ }^{2, b}$ \\ ${ }^{1}$ Hangzhou Dianzi University, Hangzhou 310018, China \\ ${ }^{2}$ Hangzhou Dianzi University, Hangzhou 310018, China \\ abxy002@126.com, ${ }^{b}$ bluerose0617@126.com
}

\begin{abstract}
Under e-business environment, the evolutionary process of channel selection of service provider and customer is researched by evolutionary game. Firstly, the evolutionary game model between the supply and the demand parties of service is built. Then the channel selection to the traditional channel and the dual-channel is analyzed. In addition, the equilibrium points are obtained. Finally, equilibrium points are analyzed to obtain evolutionary stable strategy. Research results are as follows: yield gap between network channel and traditional channel of service provider and consumer utility in both channels have influence on evolutionary stable strategy. Service provider and customers ought to take these factors into consideration when they select channels.
\end{abstract}

Keywords:Channel Selection Strategy, Evolutionary game, Service characteristics, Evolutionary stable strategy.

\section{Introduction}

A growing number of companies redesign their tradition sale channel structures by engaging in direct sale to form a dual-channel mode. Forrester research shows that China has the world's largest number of online consumers. By the end of 2014, online sales in China are expected to more than $\$ 300$ billion [1]. Moreover, iClick, indicated that per-capita consumption of online shopping was about $\$ 840$ a year, growing by $25 \%$, and was estimated to become the first in the world in 2015 [2].

Therefore, many scholars had studied the issues of channel selection. Yan used game theory to prove that the manufacturers and retailers can benefit from dual-channel strategy[3]. Cai studied the structure and coordination of dual-channel based on Pareto theory[4]. Yan and Pei also found that dual-channel supply chain can promote the service level of the retailer and increase the performance[5]. Some scholars pointed out that, engaging in direct channel may cause channel conflict. Hendershott [6] and Xiao [7] pointed out that manufacturers prefer to choose the traditional channel when facing high costs or high substitution. 
The researches mentioned above mainly studied the channel selection in manufacturing. However, the market has gradually shifted from product-oriented to service-oriented. This paper analyzes how strategies of channel selection of service provider and consumers evolve dynamically under limited rational condition by evolutionary game theory, which provides suggestions for service provider.

\section{Problem statement and assumption}

In traditional channel, there is a monopolistic service provider to provide service for customers directly. Service provider can also sell service by online distribution. However, network channel is limited by many factors. Customers' network awareness, network preferences have influence on their choices. Based on their channel preference heterogeneity, the consumers can be divided into two classes: ordinary consumers and network consumers. The assumptions are as follows.

Assumption 1. In service market, the amount of potential customers is $Q$. Network consumers account for $\delta(0<\delta<1)$, and the total number of network consumers are $\delta Q$. Accordingly, the number of ordinary consumers are $Q(1-\delta)$. And this paper assumes that $Q=1$;

Assumption 2. In traditional channel, the price of service is $p_{d}$, the cost to provide service is $C_{d}$, and general publicity expenses of service provider is $C_{a}$, thus the cost is $C_{f}=C_{d}+C_{a}$. In network channel, the price is $p_{k}$, and the agency commission service provider pays is $C_{k}$. So the cost is $C_{h}=C_{d}+C_{k}\left(C_{k}<C_{a}\right)$. In addition, the fixed costs (shop rent, etc.) are defined as $C_{e}$;

Assumption 3. Based on the consumer utility theory, we assume that the willingness-to-pay of ordinary customers purchasing service in traditional channel is $S$, and $S$ obey uniform distribution on $[0,1]$. The willingness-to-pay in network channel is $\mu$, and $\mu(0<\mu<1)$ denotes the acceptance degree of ordinary customers to network channel. Consequently, ordinary customers' net utility in traditional channel and in network channel are $U_{d}^{C}=s-P_{d} \quad$ and $\quad U_{k}^{C}=\mu s-P_{k} \quad$. And when $\quad P_{d}>\frac{P_{k}}{\mu}$, $q_{d}^{C}=(1-\delta) Q\left(1-\frac{P_{d}-p_{k}}{1-\mu}\right) \quad$ and $\quad q_{k}^{C}=(1-\delta) Q\left(\frac{P_{d}-p_{k}}{1-\mu}-\frac{p_{k}}{\mu}\right)$. So network consumers' demand are $q_{d}^{O}=0$ and $q_{k}^{O}=\delta Q\left(1-P_{k}\right)$. Thus traditional and network channel demand are: 


$$
\begin{aligned}
& D_{d}=(1-\delta)\left(1-\frac{P_{d}-p_{k}}{1-\mu}\right) . \\
& D_{k}=(1-\delta)\left(\frac{P_{d}-p_{k}}{1-\mu}-\frac{p_{k}}{\mu}\right)+\delta\left(1-P_{k}\right) .
\end{aligned}
$$

\section{Basic model analyses}

Service is inseparable, provider provides service under traditional channel. In order to facilitate analysis, this paper only considers one provider provides service for consumers, and only sells service through a single mode. And consumers have the unique channel selection. So service's demand function is $D_{d}=1-P_{d}$, and the benefit of provider is $G^{T}\left(P_{d}\right)=\left(P_{d}-C_{f}\right)\left(1-P_{d}\right)-C_{e}$. Consequently, in traditional channel, service provider's benefit is $G_{d}^{T}=\frac{\left(1-C_{f}\right)^{2}}{4}-C_{e}=A-C_{e}$.

Service provider sells service in network channel depending on service agent, and the provider will pay agency commission when the agent sells service successfully. As service's agent, its benefit links with its sales quantities, and has no direct influence on channel selection. Under dual-channel, the benefit of service provider concludes traditional channel benefit and network channel benefit.

On this occasion, the total demand of traditional channel and network channel are

$$
D_{d}=(1-\delta)\left(1-\frac{P_{d}-P_{k}}{1-\mu}\right)
$$

and

$D_{k}=(1-\delta)\left(\frac{P_{d}-P_{k}}{1-\mu}-\frac{P_{k}}{\mu}\right)+\delta\left(1-P_{k}\right)$. In addition, the benefit function of provider is $G^{D}\left(P_{d}, P_{k}\right)=\left(P_{d}-C_{f}\right) D_{d}+\left(P_{k}-C_{h}\right) D_{k}-C_{e}=B+C-C_{e}$.

\section{$4 \quad$ Evolutionary game modeling}

We assume that the percentage of service provider choosing the sales mode of dual-channel is $p(0 \leq p \leq 1)$. Similarly, the percentage of consumers choosing the consumption mode of network channel is $q(0 \leq q \leq 1)$. So the benefit matrix of evolutionary game is shown in Table 1. 
Table 1: Benefit matrix of evolutionary game.

\begin{tabular}{|c|c|c|c|}
\hline \multirow{2}{*}{\multicolumn{2}{|c|}{ Game Matrix }} & \multicolumn{2}{|c|}{ Consumers } \\
\hline & & $q$ & $1-q$ \\
\hline \multirow{3}{*}{$\begin{array}{c}\text { Service } \\
\text { Provid } \\
\text { er }\end{array}$} & \multirow{2}{*}{$p$} & $B-C_{e}$ & $C-C_{e}$ \\
\hline & & $(1-\delta)\left(\mu s-P_{k}\right)+\delta\left(s-P_{k}\right)$ & $(1-\delta)\left(s-P_{d}\right)$ \\
\hline & $1-p$ & $-C_{e}, 0$ & $A-C_{e}, s-P_{d}$ \\
\hline
\end{tabular}

From Table 1, the fitness of provider choosing dual-channel and single-channel sales strategy are:

$U^{D}=q\left(B-C_{e}\right)+(1-q)\left(C-C_{e}\right)$ and $U^{T}=q\left(-C_{e}\right)+(1-q)\left(A-C_{e}\right)$.

So average fitness is $\bar{U}=p U^{D}+(1-p) U^{T}$. Hence, the replicator dynamics formula is

$U(p)=\frac{d p}{d t}=p\left(U^{D}-\bar{U}\right)=p(1-p)[q(B+A-C)-(A-C)]$

Similarly, from the perspective of consumers, the replicator dynamics formula of consumers is

$V(q)=\frac{d q}{d t}=q(1-q)\left[p\left(\mu s+2 \delta s-P_{k}-\mu \delta s-\delta P_{d}\right)-\left(s-P_{d}\right)\right]$

From the above, we can obtain a two-dimensional dynamic system (I).

\section{Model analyses}

From the system (I), we can get five partial equilibriums: $(0,0) 、(0,1)$ 、 $(1,0) 、(1,1)$ 和 $\left(p^{*}, q^{*}\right)$, and $p^{*}=\frac{s-P_{d}}{\mu s+2 \delta s-P_{k}-\mu \delta s-\delta P_{d}}$, $q^{*}=\frac{A-C}{B+A-C}$. Jacobi matrix is used to judge above equilibriums. If $J$ satisfies two conditions ( $\operatorname{tr} J=a_{11}+a_{22}<0$; $\operatorname{det} J=a_{11} a_{22}-a_{12} a_{21}>0$ ) simultaneously, partial equilibriums are ESS. However, there is $a_{11}+a_{22}=0$ in point $\left(p^{*}, q^{*}\right)$, that is, this point doesn't meet Condition 1, so it is not ESS. Based on evolutionary game matrix, this paper will analyze the possibility of the four partial equilibriums become ESS under different conditions. 


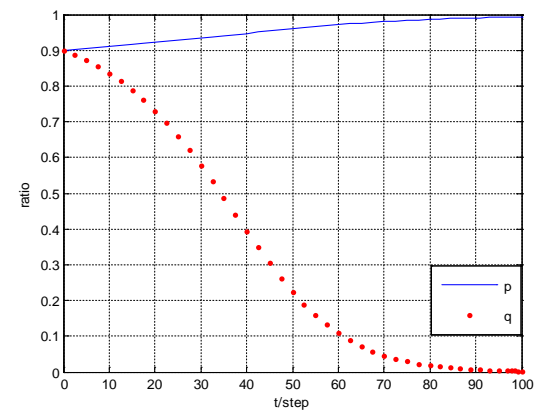

Fig.1: Evolutionary trend of situation 1

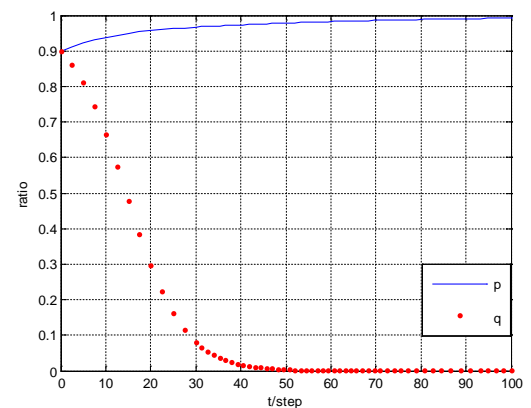

Fig.2: Evolutionary trend of situation 2

$$
\text { (1)When } \quad C-A>0 \quad, \quad s-P_{d}<0
$$

$\mu s+2 \delta s+P_{d}-P_{k}-\mu \delta s-\delta P_{d}-s<0 \quad, \quad$ then, $\quad(1,0) \quad$ is ESS, $(0,1)$ and $(1,1)$ are saddle points, $(0,0)$ is unstable point, the evolution trend is shown in Fig.1.

Service provider can achieve good promotional effect by taking advantage of the agent, that is, $C_{a}$ decreases, then the net income of the service provider taking the dual-channel sales mode is higher than traditional channel sales mode. But for consumers, network just shows the description of services. Consumers will search for information online, and buy from traditional channel.

$$
\text { When } \quad C-A>0
$$$$
s-P_{d}>0
$$

$\mu \mathrm{s}+2 \delta s+P_{d}-P_{k}-\mu \delta s-\delta P_{d}-s<0 \quad, \quad$ then, $\quad(1,0) \quad$ is ESS, $(0,0)$ and $(1,1)$ are saddle points, $(0,1)$ is unstable point, the evolution trend is shown in Fig.2.

Service provider gets more benefits from network channel, so the net income of dual-channel sales mode is greater than traditional mode, provider will choose dual-channel. Due to the popularity of network, consumers are used to searching online before consumption. Thus when the price difference between two channels is low, consumers prefer traditional channel consumption.

(3)When

$$
C-A<0
$$

$$
s-P_{d}>0
$$

$\mu s+2 \delta s+P_{d}-P_{k}-\mu \delta s-\delta P_{d}-s<0 \quad$, then, $(0,0)$ is ESS, $(1,0)$ and $(1,1)$ are saddle points, $(0,1)$ is unstable point, the evolution trend is shown in Fig.3.

The income service provider gets from network is low and the net income of dual-channel is smaller than that of traditional channel, provider selects the traditional mode. And consumer utility in dual-channel mode is lower than that in traditional mode. Consumers prefer purchasing service from traditional channel. 
(4)When $C-A<0, s-P_{d}>0, \mu s+2 \delta s+P_{d}-P_{k}-\mu \delta s-\delta P_{d}-s>0$, then, $(1,1)$ is ESS, $(0,0)$ and $(0,1)$ are saddle points, $(1,0)$ is unstable point, the evolution trend is shown in Fig.4.

The benefit of provider taking network channel is less than traditional channel, but the net income gained from dual-channel is higher than that from traditional channel, provider will select dual-channel mode to charm more consumers and improve market share. And service's price in network channel is more attractive, so consumers will choose the network consumption pattern.

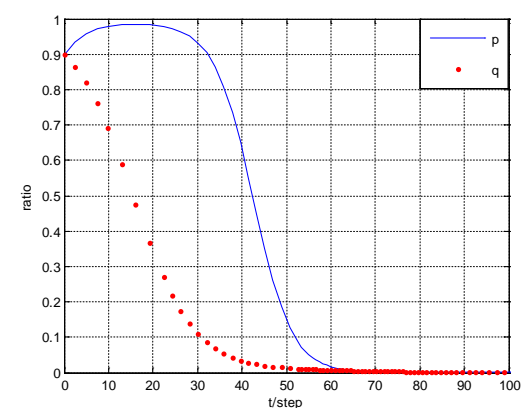

Fig.3: Evolutionary trend of situation 3

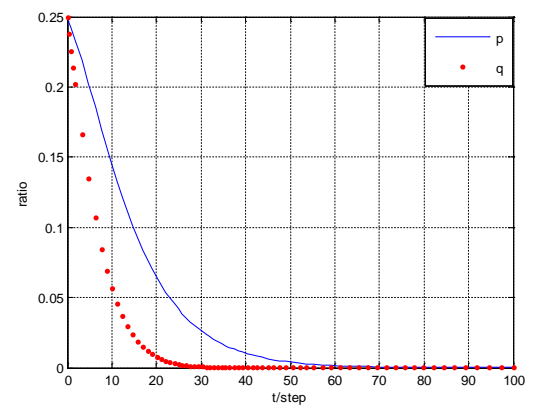

(a)

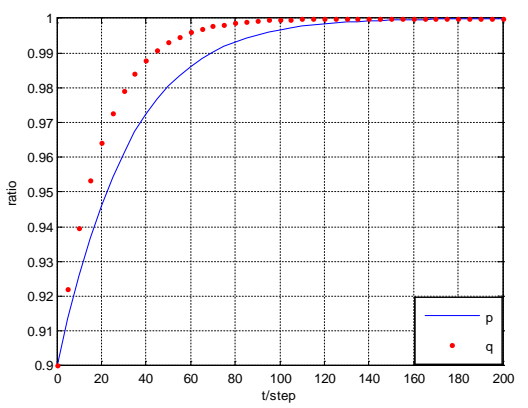

Fig.4: Evolutionary trend of situation 4

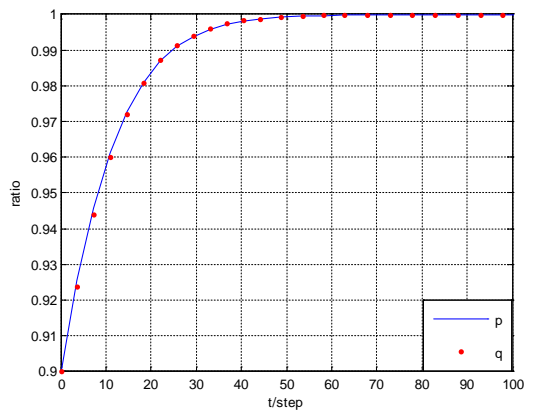

(b)

Fig.5: Evolutionary trend of situation 5 When $C-A<0$ $S-P_{d}>0$ $\mu s+2 \delta s+P_{d}-P_{k}-\mu \delta s-\delta P_{d}-s>0$, then, $(0,0)$ and $(1,1)$ are ESS, $(0,1)$ and $(1,0)$ are unstable points, which are shown in Fig. 5.

If $C<A$, it can't obtain the result that the net income of dual-channel sales mode is less than that of traditional mode. Then, stable strategy depends on the initial state of the system. Fig.5 (a) shows: if consumers choosing traditional 
channel have a large percentage, consumers will purchase service such as consulting, etc. through traditional channel. Consumers are willing to making purchase decision after face-to-face communication. Fig.5 (b) shows: if more consumers choose network mode, consumers will purchase service such as movies, etc. through network. Despite provider gets lower gains, he still can choose dual-channel sales mode to attract network consumers.

(6)When $C-A>0, s-P_{d}>0, \mu s+2 \delta s+P_{d}-P_{k}-\mu \delta s-\delta P_{d}-s>0$, then, $(1,1)$ is ESS, $(0,0)$ and $(1,0)$ are saddle points, $(0,1)$ is unstable point, the evolution trend is shown in Fig.6.

Net income of taking dual-channel mode is higher than that of traditional mode, provider will select dual-channel sales mode. Also, provider can control the cost and provide more favorable prices for consumers, so consumer utility in both channel strategies is greater than zero. Then the price difference become the main factor that influences the consumers' decision, and when $P_{d}>P_{k}$, consumers will choose the network consumption pattern.

$$
\text { When } \quad C-A>0
$$

$s-P_{d}<0, \mu s+2 \delta s+P_{d}-P_{k}-\mu \delta s-\delta P_{d}-s>0$, then, $(1,1)$ is ESS, $(0,1)$ and $(1,0)$ are saddle points, $(0,0)$ is unstable point, the evolution trend is shown in Fig. 7.

Net income of service provider adopting dual-channel mode is higher than traditional channel mode, provider will select dual-channel mode. For traditional sales mode, provider needs to invest lots of publicity expenses, then $C_{a}$ increases, and the cost maybe passed on to consumers, then $P_{d}$ increases, which decreases the consumer utility and more consumers select purchase online.

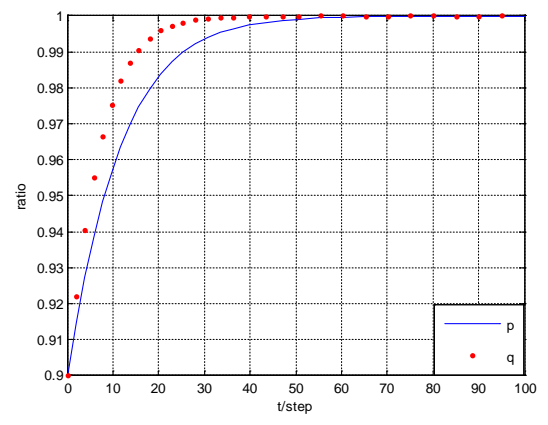

Fig.6: Evolutionary trend of situation 6

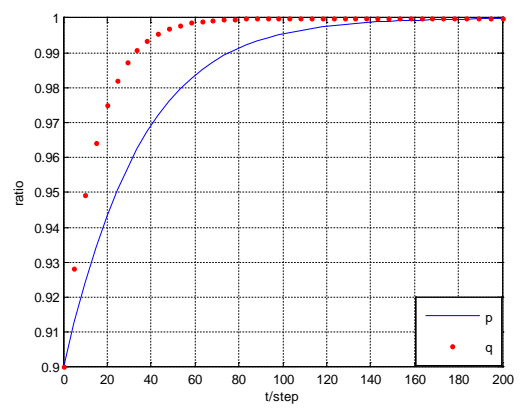

Fig. 7: Evolutionary trend of situation 7

$$
\text { (8)When } \quad C-A<0 \quad, \quad s-P_{d}<0
$$

$\mu s+2 \delta s+P_{d}-P_{k}-\mu \delta s-\delta P_{d}-s<0$, then, there is no ESS. Service provider gets fewer benefits from network mode than traditional mode, and 
consumer utility is less than zero. Provider and consumers don't tend to stable points.

From above, the results can be concluded that channel selection of service provider and consumers has three steady strategies: $(0,0),(1,0)$ and $(1,1)$.

\section{Conclusions}

Under the rapid development of e-commerce, service provider has to consider whether to develop network distribution channel. The results are as follows.

(1) If the benefit of service provider from network channel is less than traditional channel, and consumers' utility in traditional channel is greater than zero, both of them will choose traditional channel mode. Thereby, only when network sales channel becomes ripe, can service provider get more profit from it by dual-channel strategy, and can consumers benefit from it.

(2) If the benefit of service provider from network channel is greater than traditional channel, and consumers' utility in dual-channel is less than zero, service provider will choose dual-channel sales mode and consumers will choose traditional channel consumption pattern. On this occasion, network channel is regarded as a promotional channel.

(3) If consumers' utility in dual-channel is greater than zero, service provider will choose dual-channel sales mode and consumers will choose network consumption pattern.

\section{Acknowledgements}

This work was financially supported by the Zhejiang province philosophy social sciences planning project (13NDJC038YB).

\section{References}

[1] Kelland W, Zia D W. China Online Retail Forecast, 2013 To 2018. Forrester Research, 2014.

[2] Information on: http://www.i-click.com/sc/

[3] Yan R. Profit sharing and firm performance in the manufacturer-retailer dual-channel supply chain. Electronic Commerce Research, Vol. 8(3), 2008, p. 155-172.

[4] Cai G G. Channel selection and coordination in dual-channel supply chains. Journal of Retailing, Vol. 86(1), 2010, p. 22-36.

[5] Yan R, Pei Z. Retail services and firm profit in a dual-channel market. Journal of Retailing and Consumer Services, Vol. 16(4), 2009, p. 306-314.

[6] Hendershott T, Zhang J. A model of direct and intermediated sales. Journal of Economics \& Management Strategy, Vol. 15(2), 2006, p. 279-316.

[7] Xiao T, Choi T M. Purchasing choices and channel structure strategies for a two-echelon system with risk-averse players. International Journal of Production Economics, Vol. 120(1), 2009, p. 54-65. 